\title{
Pre-filled Syringe
}

National Cancer Institute

\section{Source}

National Cancer Institute. Pre-filled Syringe. NCI Thesaurus. Code C97716.

A syringe designed to dispense a predetermined dose of a drug. A prefilled syringe has the advantage of not requiring mixing or transfer of drug between containers. Most prefilled, single use syringes resemble a standard syringe but those designed to deliver several doses may resemble a writing pen. 